\title{
Percepción de consumidores y expertos sobre los valores de la publicidad del sector del automóvil (2008-2018)
}

\section{The perception of consumers and experts regarding the values of advertising in the automotive sector (2008-2018)}
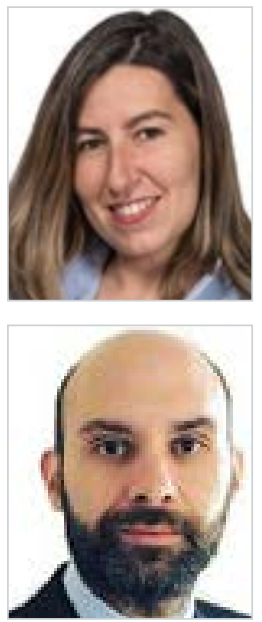

Recibido: 06/06/2021 - Aceptado: 19/09/2021 - En edición: 01/12/2021 - Publicado: 01/01/2022

\section{Resumen:}

Cada día los productos son más similares y se diferencian menos unos de otros y, con la globalización, la competencia entre ellos es aún mayor. La publicidad es uno de los factores de diferenciación más relevantes para que los consumidores elijan unos u otros en el momento de su decisión de compra. Como consecuencia de este fenómeno y de los peculiares y complejos factores que parecen determinarlo, en este trabajo se pretende estudiar y analizar la escala de valores subyacente por los que una persona elige un automóvil en la actualidad y el papel que la publicidad ejerce en esa decisión. Esta investigación analiza los valores transmitidos en la publicidad efectuada en Internet por la industria automovilística. Para ello, se ha realizado una comparación entre los valores de la publicidad durante 2008 y 2018 respecto
Received:06/06/2021 - Accepted: 19/09/2021 - Early access:01/12/2021 - Published:01/01/2022

\section{Abstract:}

Products are becoming increasingly similar, and now it is more difficult to distinguish one from the other. Moreover, as a result of globalisation, the competition between them is more intense as well. Advertising is one of the most significant differentiating factors for consumers in choosing one product over another when they make a purchase decision. Given this phenomenon, as well as the complex and peculiar factors that seem to determine the situation, this paper aims to study and analyse the underlying scale of values by which a person chooses a car today, and the role that advertising plays in that decision. The research herein analyses the values communicated through Internet adverts by the automobile industry. To this end, a comparison has been made between the values of advertising that appeared in 2008

Cómo citar este artículo:

Bonales Daimiel, G. y Mañas-Viniegra, L. (2022). Percepción de consumidores y expertos sobre los valores de la publicidad del sector del automóvil (2008-2018). Doxa Comunicación, 34, pp. 155-176.

https://doi.org/10.31921/doxacom.n34a894 
a los criterios y valores de los consumidores en la toma de decisión de compra de un coche y los valores que, según los expertos, son clave en el proceso de compra. Se ha utilizado una metodología mixta cuantitativa-cualitativa para identificar la percepción de 300 consumidores y 30 expertos y los valores transmitidos por la publicidad a través del análisis de contenido de 672 anuncios. Los resultados muestran que hay una divergencia relativamente clara entre los valores que la publicidad transmite, los que demandan los clientes y los que los expertos consideran más relevantes.

\section{Palabras clave:}

Valores; automóvil; publicidad; consumidores; comunicación. and 2018 with regard to the criteria and values of consumers when making an automobile purchase decision, as well as the key values involved in the purchasing process, according to experts. A combined quantitative-qualitative methodology has been used to identify the perceptions of 300 consumers and 30 experts, as well as the values conveyed by advertising through a content analysis of 672 adverts. The results show that there is a relatively clear divergence between the values that advertising communicates, the ones that customers demand, and those that experts consider most significant.

\section{Keywords:}

Values; automobile; car; advertising; consumers; communication.

\section{Introducción}

Los valores ocupan un espacio esencial en el ámbito de la Psicología y la sociedad, pues son el resultado de cambios y transformaciones de la historia (Sandoval, 2007). Son las creencias abstractas y estables que trascienden situaciones específicas, se ordenan jerárquicamente en términos de importancia y se utilizan para resolver conflictos o tomar decisiones (Schwartz y Bilsky, 1990; Barbarossa et al., 2017). Rodríguez et al. (2013) estudian los valores desde tres dimensiones: la educativa, la humana y la social, definiéndolos como

Aquellos elementos de sus contenidos y aquellos rasgos de sus características formales que configuran el conjunto de cualidades que otorgan importancia, validez o mérito a una producción comunicativa, haciéndola ser apreciada por su adaptación a las necesidades del hombre y la sociedad (Rodríguez et al., 2013, p. 11).

El empleo de valores en las estrategias publicitarias no es una práctica innovadora. "Desde la pre-publicidad hasta la publicidad, sus discursos han supuesto un eficaz diagnóstico de los valores sociales y culturales que han imperado en cada época" (Núñez-Cansado y Martín-Requero, 2015, p.39). Tanto los valores colectivos -juventud, modernidad, tecnología o tradicióncomo los valores transitivos -amistad, solidaridad o ecología- han constituido la base de las campañas publicitarias, permitiendo la creación de atribuciones de nuevas simbologías al producto y/o marca que nunca podrían haber encarnado por sí mismos.

Ya casi nadie duda, por ejemplo, de que los argumentos y la práctica publicitaria tienden a sustituir los atributos de los productos por todo un conjunto de valores intangibles derivados de los rasgos de imagen de la organización o de los aspectos muy personalizados de los consumidores (Victoria, 2005, p. 16).

Desde esta perspectiva, la publicidad debe contemplarse, por un lado, como elemento sociológico con vocación económica que se renueva a la par que lo va haciendo el mercado y, por otro, como "un factor socializador [que] se convierte en una representación cultural” (González Requena y Ortiz de Zárate, 1999, citado en Ramos, Delgado y Jiménez, 2007, p. 32). 


\subsection{La creación de valores a partir de los medios de comunicación y la publicidad}

Los medios han marcado la evolución de la publicidad, y esta a su vez ha permitido la revolución de los medios; pero ningún medio anterior ha supuesto un cambio tan rápido en el panorama comunicativo como lo está haciendo actualmente Internet (MartínCasado et al., 2012, p. 165).

Tradicionalmente, la televisión ha sido citada como el medio más relevante por el público en general, hasta el punto de asignarle el mérito de haber cambiado el mundo de la comunicación (Gunter et al., 2000). Al haber superado a otros medios en su capacidad para atraer la atención de los espectadores (Johnson y Kaye, 2000), los anunciantes han destinado una mayor inversión a la televisión históricamente, lo que supuso que otros medios comenzaran su declive. No solo el cine, sino también las revistas recibieron un duro golpe de este -entonces- nuevo medio (McLuhan, 1994, p. 318), permitiendo al espectador ver los mensajes publicitarios con imagen y sonido, facilitando el recuerdo y que todo aquello que se anunciaba en televisión tuviera un elevado retorno.

Sin embargo, el crecimiento de la inversión publicitaria en Internet ha sido el mayor de todos los medios desde finales de 2016, en especial por parte de los anunciantes del sector del automóvil. Así, Internet ha crecido un $21 \%$ y en el sector automoción aumentó un 75\% desde 2017 (Infoadex, 2018). Este soporte digital es mucho más que una tecnología, "es un medio de comunicación, de interacción y de organización social" (Castells, 2006). Ha constituido un nuevo metamedio que ha provocado que el resto de medios de comunicación hayan tenido que adaptarse (Okazaki, 2002).

En este sentido, la publicidad que se desarrolla en Internet ha sido etiquetada como Electronic Advertising y presenta diferencias importantes con la tradicional, como es la interactividad con respecto a la publicidad realizada en otros medios de comunicación (Hawkins, 1994; Yoon y Kim, 2001).

Por su parte, la publicidad transmite valores como el respeto al medio ambiente, la no violencia, la felicidad familiar e, incluso, la solidaridad. Hay ocasiones en las que se llegan a omitir las características del producto, pues el objetivo principal de esta es alcanzar una imagen de marca positiva.

El mensaje publicitario, por medio de la persuasión y la interpelación a sentimientos y emociones, dialoga no sólo con las necesidades, sino también con el aparato cultural de valores, hasta el punto de que se le otorga mayor protagonismo al valor de marca de un producto que a sus atributos intrínsecos (García, 2018, p. 94).

La publicidad puede entenderse como un actor que transmite al unísono significados y valores: primero, al producto y, en segundo lugar, desde el producto a los públicos. Es así como la publicidad esteriliza el producto, porque lo transforma en atributos deseables para el consumidor que se transfieren a este a través de la compra y que, a su vez, lo hacen deseable frente a los demás por poseer el producto. "Este proceso es factible gracias a la participación del consumidor, que actúa de forma activa como intérprete de los valores y significados asociados a los productos y servicios y no de forma pasiva” (Coldeluppi, 2007, p.151).

\subsection{Los modelos de valores}

Autores como Pollay (1986); Tse et al. (1989); Schwartz (1994); Domínguez (1995) o Núñez-Cansado y Martín-Requero (2015) han creado o utilizado modelos y construcciones teóricas existentes para definir los factores que cobran protagonismo en la toma 
de decisión de compra de un producto y, en consecuencia, en la publicidad. No obstante, son pocos los que han aplicado esta evaluación de valores a los automóviles.

En la teoría de modelos para la revisión de valores de marca, la mayoría de los estudios emplea una muestra de anuncios de prensa y/o televisión y tiene su origen en el modelo establecido por Pollay (1983), quien identificó los valores dominantes de la publicidad y los categorizó. Pollay (1986) entendía la publicidad como un metáfora -"espejo cultural distorsionado"- donde solo se reflejan ciertas actitudes, comportamientos y valores, aquellos que sirven a los intereses del vendedor por estar más fácilmente vinculados a los productos disponibles, que se dramatizan fácilmente en los anuncios y a los que los consumidores que ven los anuncios responden de manera más confiable (Okazaki, 2002).

Pollay analizó más de 400 anuncios de tres países distintos y estableció inferencias de valor entre los anuncios de las mismas categorías de producto en esos países, apoyándose en una tabla que abarcaba 42 valores, de los cuales destacan la practicidad, la tecnología, lo natural, la economía, la productividad, la juventud, el estatus social o la pertenencia, entre otros. Así, Pollay concluyó que había "una gran consistencia en el tiempo y en los distintos medios" acerca de los valores más frecuentemente promovidos en el discurso publicitario (Méndiz, 2007). Por ello, la consecuencia final de la publicidad es la consolidación de valores negativos como el materialismo, el cinismo, la ansiedad, la competitividad social y la ausencia de respeto.

Posteriormente, Tse et al. (1989), siguiendo la propuesta de Pollay, realizaron una estandarización de los valores de los consumidores adaptada a las particularidades asiáticas (Tabla 1). Para ello, emplearon una muestra consistente en 291, 296 y 377 anuncios de prensa seleccionados desde el año 1979 hasta 1985. Las categorías -llamamientos de consumo- empleadas en el análisis de contenido cultural que realizaron estos autores fueron las siguientes:

Tabla 1. Llamamientos de consumo

\begin{tabular}{|l|l|}
\hline \multicolumn{1}{|c|}{ Llamamientos de consumo } & \multicolumn{1}{c|}{ Componentes listados en el formulario de codificación } \\
\hline Tecnología & Contenido tecnológico del producto \\
\hline Modernidad & Moderno, futuro, nuevo \\
\hline Hedonismo & Bonito, lujo, prestigio, extranjero, divertido \\
\hline Comportamiento del producto & Alta calidad, comportamiento \\
\hline Garantía del producto & Certificado, generalmente aceptado \\
\hline Ingredientes & Ingredientes del producto \\
\hline Variedad del producto & Variedad \\
\hline Distribución & Disponibilidad del servicio, lista de tiendas o talleres \\
\hline Valor de la compra & Precio, economía, práctico \\
\hline Imagen & Historia empresarial, imagen \\
\hline & Fuente: Tse et al (1989) \\
\hline
\end{tabular}

Schwartz (1994) también estudió los factores que influyen en la decisión de compra y estableció una Encuesta de Valor (SVS) en la que definía diez tipos de valores según el tipo de objetivos motivacionales que expresan, estructurándolos de manera circu- 
lar (Figura 1) para enfatizar sus relaciones de similitud frente al conflicto. Schwartz los organizó en cuatro dominios de orden superior:

- "Auto-trascendencia", que combina benevolencia y universalismo. La benevolencia indica preocupación por el bienestar de los demás y el universalismo enfatiza la comprensión y la protección del interés de todas las personas y la naturaleza.

- "Auto-mejora" es el resultado del poder y el logro. El poder indica control y dominio sobre personas y recursos, estatus social y prestigio; mientras que el logro se refiere al éxito y la aprobación social.

- "Apertura al cambio" integra la autodirección, la estimulación y los tipos de valor hedónico, enfatizando la gratificación sensual y el placer para uno mismo.

- "Conservación" incluye la seguridad, la conformidad y la tradición. Esta última, lleva a las personas a priorizar las reglas sociales establecidas y las expectativas inmutables del pasado (Barbarossa et al., 2017).

Figura 1. Valores de Schwartz para los dominios de valor

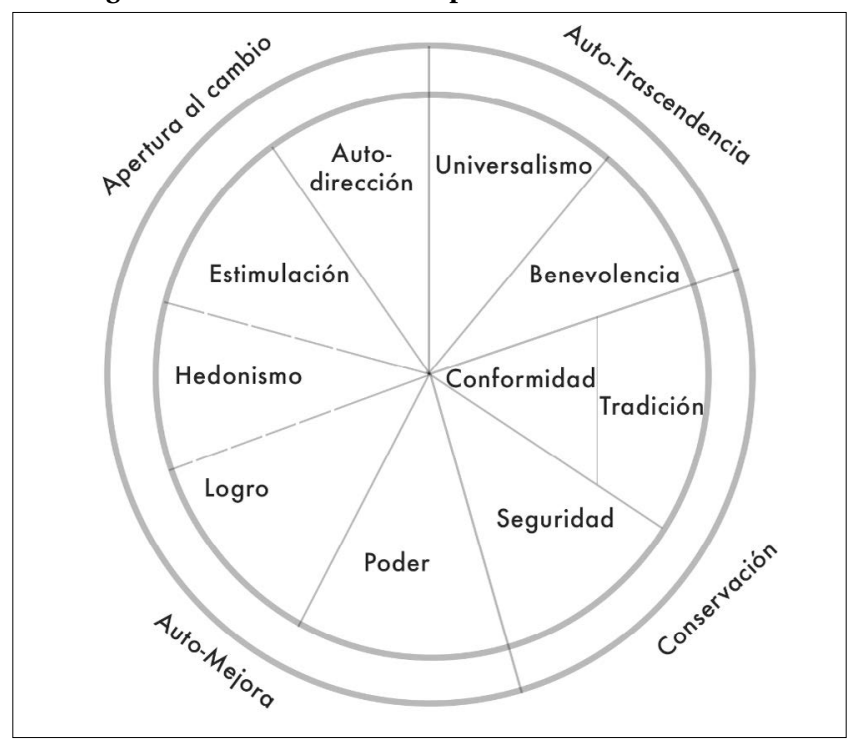

Fuente: elaboración propia a partir de Barbarossa et al. (2017)

Posteriormente, Domínguez (1995) llevó a cabo un análisis detallado de los valores implícitos en la publicidad televisiva española sobre una muestra de 500 anuncios seleccionados a lo largo de un año. El esquema metodológico ofrecido por esta presenta grandes núcleos conceptuales que se articulan en torno a tres grandes grupos: los valores del yo, que agrupan las tendencias egocéntricas de la persona y que están centradas en el éxito, el placer, la comodidad o la seguridad; los valores colectivos, que son los que nos unen a los demás, al grupo; y los valores transitivos, que implican salir de uno mismo para abrirse solidariamente a los demás, como son la amistad, la convivencia o el servicio. 
En los últimos años, autores como Alameda (2006), Núñez et al. (2008) o Castelló et al. (2013) consideran que la publicidad, más que transmitir los valores de un país, refleja los valores materialistas que los anunciantes transmiten. Núñez-Cansado y Martín-Requero (2015) también identifican la existencia de estos valores materialistas (altos ingresos, crecimiento, orden, seguridad) y señalan el cambio de estos hacia valores post-materialistas (participación, equilibrio ecológico...) como el resultado de una "revolución silenciosa" en los países industrializados. Según estos, el tránsito de los viejos valores hacia los post-materialistas ha generado consecuencias, como la aparición de tendencias ecologistas que conllevan la repentina preocupación por el medio ambiente (Núñez-Cansado y Martín-Requero, 2015, p. 39).

\subsection{Modelos aplicados al sector del automóvil y valores influyentes en la decisión compra}

Para la mayoría de los consumidores, comprar un automóvil nuevo es un comportamiento esporádico por ser una decisión de compra con alta implicación. Consecuentemente, en comparación con otras muchas decisiones de compra regulares y de menor escala para las que el hábito puede ser importante, puede haber una mayor probabilidad de procesos de pensamiento racional que generan un análisis coste-beneficio (Steg, 2005; Lieven et al., 2011; Gao et al., 2014; Hafner et al., 2017).

Las influencias contextuales más específicas incluyen consideraciones utilitarias, como el precio de compra, el tamaño, el rendimiento y los gastos de funcionamiento (Lane y Potter, 2007; Banerjee, 2010), así como cuestiones más relacionadas con la imagen -por ejemplo, el color- y el estado -la marca-, que están vinculadas a preocupaciones normativas y de identidad (Choo y Mokhtarian, 2004; Peters et al., 2015). También existe evidencia de que el impacto ambiental de la compra de un automóvil, por ejemplo, las emisiones de $\mathrm{C}_{\mathrm{O} 2}$ y partículas, es una consideración de interés (Kahn, 2007; Coad et al., 2009). La conducción de un determinado vehículo tiene una serie de connotaciones más allá de los propios beneficios intrínsecos de transporte, como las asociaciones simbólicas que proporcionan y expresan una identidad propia y un determinado estatus social (Steg et al., 2001; Steg, 2005; Heffner et al., 2007). Estos beneficios relacionados con la propia imagen social pueden ser tenidos en cuenta, incluso, por encima de otros más racionales relacionados con los beneficios intrínsecos del vehículo en el momento de la decisión de compra (Lane, 2011). "Los beneficios simbólicos influencian las decisiones de compra de los clientes, y pueden ser incluso creadores de tendencias o modas en las ventas" (Burgess et al., 2013, p. 33).

De esta forma, las marcas emplean una estrategia diferente en función de sus modelos y del target al que quieren dirigirse, ya que estas dependen de los distintos atractivos que tiene cada vehículo (Heffner et al., 2005; Burgess et al., 2013).

En cuanto al estudio de la decisión de compra, existe el proceso jerárquico analítico o analytic hierarchy process-AHP (Figura 2) establecido por Byun (2001), donde se presenta un método de toma de decisiones para seleccionar los mejores modelos de automóviles de pasajeros mediante tres niveles. 
Figura 2. Modelo AHP

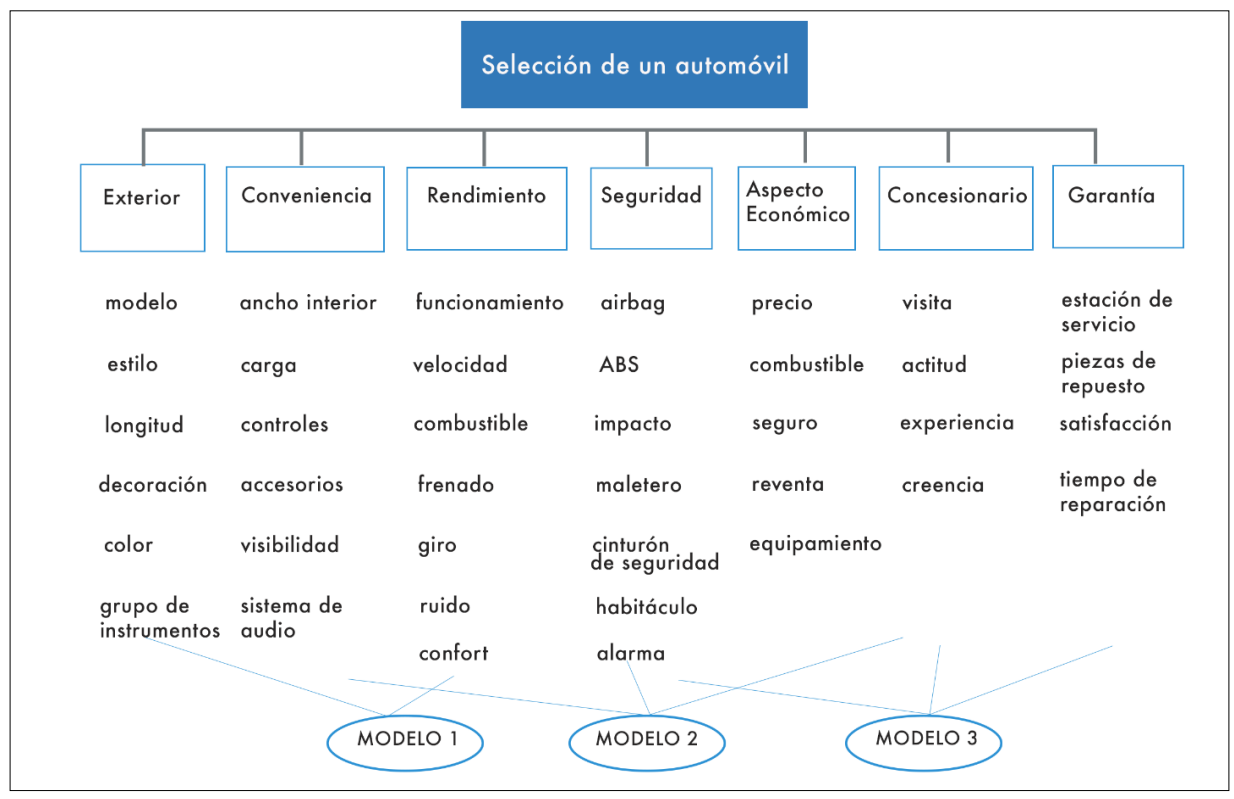

Fuente: Byun (2001)

Este método establece siete criterios en un primer nivel -el exterior, la conveniencia, el rendimiento, la seguridad, los aspectos económicos, el concesionario y la garantía- y, de estos, emergen otros factores o subcriterios, en un segundo nivel, como pueden ser el modelo o el estilo en el caso del exterior; la conveniencia relacionada con el diseño de los equipos para una mejor operatividad; el rendimiento vinculado con el funcionamiento del automóvil; la seguridad con el airbag o el cinturón de seguridad, características técnicas que reducen el riesgo de muerte o lesiones graves. El aspecto económico se refiere al precio y al gasto de un automóvil. El criterio del concesionario alude a las características y actitudes personales que llevan al cliente a tomar la decisión de compra. En último lugar, el factor de la garantía considera el servicio postventa, la facilidad de adquirir repuestos o la satisfacción del cliente posterior a los servicios prestados.

Otro modelo aplicado en el sector de la automoción (Tabla 2) es el elaborado por López et al. (2002), quienes se basan en las variables que mayor puntuación reciben de los consumidores en encuestas para establecer indicadores como factores clave en la compra de un automóvil. 
Tabla 2. Índices de satisfacción del consumidor

\begin{tabular}{|l|l|l|}
\hline \multicolumn{1}{|c|}{ Carrocería } & \multicolumn{1}{c|}{ Mecánica } & Sconomía \\
\hline Diseño/Estética & Motor & Consumo \\
\hline Diseño/Funcionalidad & Cambio & Coste de mantenimiento \\
\hline Acabado & Velocidad & Relación valor/precio \\
\hline Seguridad & Aceleración/Recuperación & Precio de reventa \\
\hline Habitabilidad & Dirección & \\
\hline Maletero & Frenos & \\
\hline Punto de conducción & Comportamiento/Estabilidad & \\
\hline Visibilidad e iluminación & Fiabilidad & \\
\hline Equipamiento & & \\
\hline Confort & & \\
\hline Nivel sonoro & & \\
\hline Ventilación y calefacción & & \\
\hline
\end{tabular}

Fuente: López et al. (2002)

Finalmente, el modelo de análisis de Chng et al. (2019) está basado en autores como Bamberg y Möser (2007) para estudiar los determinantes psicosociales del comportamiento proambiental y poder establecer tres factores con quince subapartados (Tabla 3) a tener en cuenta en la toma de decisión de compra de los automóviles.

Tabla 3. Variables consideradas en la compra de automóviles

\begin{tabular}{|l|l|ll|}
\hline Componente & Items & \multicolumn{2}{|c|}{ Varimax } \\
\hline Conciencia de & Motor grande & .798 & \\
imagen & Velocidad & .789 & \\
& Navegación & .680 & \\
& Estilo/diseño & .663 & \\
& Comodidad & .523 & \\
& Varianza & .25 & .763 \\
\hline Utilidad & Confiabilidad & & .705 \\
& Seguridad & & .668 \\
& Costo/impuestos/seguro & & .556 \\
& Funcionalidad/espacio interior & & .20 \\
\hline Varianza & & \\
\hline Medioambiental & Eléctrico & & .858 \\
& Amigable con el ambiente & & .505 \\
& Motor pequeño & & .13 \\
\hline & Varianza & & \\
\hline
\end{tabular}

Fuente: Chng et al. (2019) 


\section{Metodología}

El propósito tradicional de la publicidad es tener participación en los procesos y decisiones de compra, influyendo en las preferencias y comportamientos de los consumidores. De este modo, se consolidan determinados valores que se convierten en la representación de cada marca.

Desde esta orientación, la presente investigación tiene como objetivo general identificar los factores subyacentes que proyectan los anuncios en Internet del sector automotriz en 2008 y 2018, es decir, antes y después de la crisis económica.

Los objetivos específicos son:

- Identificar los valores proyectados por la publicidad del sector de la automoción.

- Determinar la percepción de esos valores desde tres perspectivas: publicidad, consumidor y experto.

- Proponer un modelo funcional para estudiar los criterios percibidos en la publicidad.

Para la realización de esta investigación, se utilizó una triangulación metodológica con enfoque cualitativo y cuantitativo.

La primera fase consistió en el análisis de contenido de 672 anuncios en Internet obtenidos de forma aleatoria y proporcional de Mosaico, la herramienta de acceso a la información cualitativa de Infoadex. Para seleccionar la muestra se siguieron los siguientes criterios de búsqueda (Figura 3).

Figura 3. Criterios de búsqueda de las creatividades en Mosaico

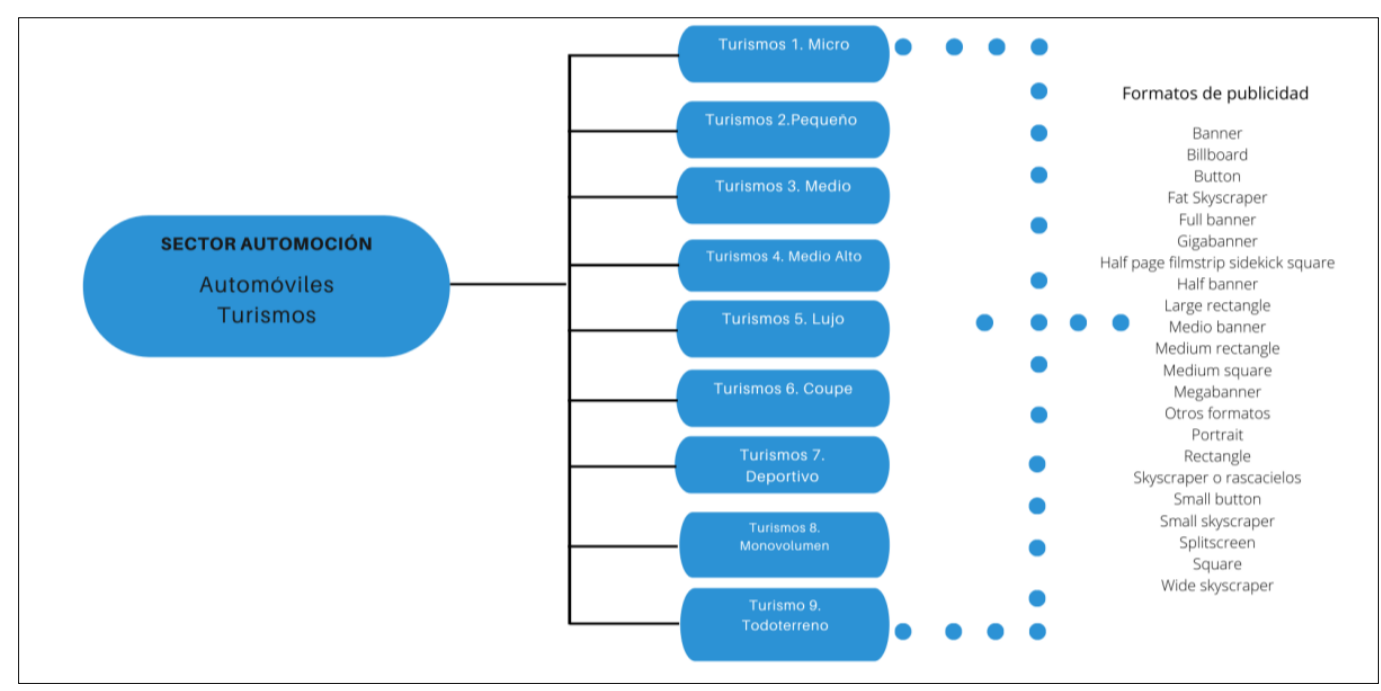

Fuente: elaboración propia a partir de Infoadex (2019) 
Con el objetivo de dimensionar apropiadamente el tamaño de la muestra de anuncios (Tabla 4) a analizar, se aplicó el algoritmo estadístico denominado $z$-score, utilizándose un nivel de confianza del 99\% y un margen de error del $5 \%$.

\section{Tabla 4. Selección de la muestra}

\begin{tabular}{|c|c|c|c|c|}
\hline Año 2008 & 1C & 2C & 3C & Total año \\
\hline No creatividades & 338 & 632 & 393 & $\mathbf{1 . 3 5 3}$ \\
\hline Muestra & 75 & 139 & 86 & $\mathbf{3 0 0}$ \\
\hline Año 2018 & $\mathbf{1 C}$ & $\mathbf{2 C}$ & $\mathbf{3 C}$ & Total año \\
\hline No creatividades & 2.688 & 5.115 & 3.156 & $\mathbf{1 0 . 9 5 9}$ \\
\hline Muestra & 91 & 174 & 107 & $\mathbf{3 7 2}$ \\
\hline
\end{tabular}

Fuente: elaboración propia

Para el análisis de las creatividades, se revisó la literatura relacionada con el estudio de la imagen, el texto y los valores. Se estudiaron y compararon los parámetros establecidos por varios autores, aunque finalmente se empleó una categorización propia. La siguiente lista muestra la ficha completa de muestreo que se usó para el análisis de las creatividades realizadas por las marcas automovilísticas en Internet, afectando a la parte de la investigación aquí presentada en la última categoría de análisis sobre los valores.

- Fecha de emisión.

- Anunciante.

- Marca.

- Modelo.

- Producto [turismos 1 micro, 2 pequeño, 3 medios, 4 medio alto, 5 lujo, 6 coupe, 7 deportivo, 8 monovolumen, 9 todoterreno].

- Tipo de banner [animación flash, jpg, gif animado].

- Formato [banner, billboard, cortinilla, layer, medio banner, megabanner, portrait, pushdown, rascacielos, robapáginas, sidekick, slider, splitscreen, superbanner].

- Medio.

- Fotogramas $[1,2,3,4,5,6,7,8,9,10,11,12,13,14,15 \ldots 20]$.

- Dimensiones [250x250, 300x250, 300x600, 468x60, 234x60, 234x90, 728x90, 900x90, 120x600, 800x600, 400x400, 900x250, 300x1050].

- Técnica [fotografía, dibujo, fotografía y dibujo, texto].

- Coche [sí, no].

- Protagonismo [producto, marca, personaje].

- Plano [Primer Plano, Plano Medio, Plano General]. 
- Fondo [neutro, ciudad, montaña, mar, carretera].

- Color fondo [blanco, gris, negro, azul, verde, naranja, rojo, amarillo, morado y marrón].

- Color coche [blanco, gris, negro, azul, verde, naranja, rojo, amarillo].

- Iluminación [artificial, natural, oscuridad].

- Titular [sí, no].

- Copy [sí, no].

- Cierre [sí, no].

- Logotipo [sí, no].

- Call to action [sí, no].

- Aviso legal [sí, no].

- Valor [seguridad, precio, financiación, promoción, garantía, ecología, tecnología, confort, espacio maletero, diseño, consumo, potencia, infoentretenimiento, prestaciones, que cumpla legislación].

2. Después, en una segunda fase para conocer la perspectiva sobre valores que tienen los usuarios, se aplicó una encuesta a 300 consumidores de automóviles con edades comprendidas entre 18 y 65 años en el ámbito nacional español, empleando la técnica de muestreo de bola de nieve. Las encuestas se llevaron a cabo desde el mes de octubre de 2019 hasta el mes de marzo de 2020 .

3. En una tercera fase, se hizo un estudio cualitativo a través de entrevistas en profundidad realizadas a 30 expertos del sector. La entrevista es una de las técnicas más utilizadas en el estudio del comportamiento del consumidor. En ella intervienen el entrevistador -personaje amistoso que establece un ambiente de confianza y libertad-, el guion, donde se planifican las preguntas -normalmente abiertas para enriquecer la información obtenida referente a las opiniones, actitudes, motivaciones, creencias y sentimientos ocultos del entrevistado- y el entrevistado, el personaje objeto de estudio (Trespalacios et al., 2005).

Para poder seleccionar a estos, se acude a una red social profesional y a visitas a puerta fría a concesionarios de la ciudad de Madrid. A algunos se les realizó la entrevista telefónica y a otros, presencialmente, con una duración aproximada de 35-45 minutos. Estos expertos fueron seleccionados en base a 3 criterios de triangulación:

- Criterio 1: Personal directivo de las principales compañías automovilísticas de España.

- Criterio 2: Periodistas del motor pertenecientes a los principales sitios webs y blogs de coches.

- Criterio 3: Fuerza de ventas. Personal de distintos concesionarios de España.

\subsection{Modelo propuesto para el análisis de valores en la publicidad automotriz}

La categorización de los valores empleados en la publicidad es otro paso esencial en esta investigación. En un principio, estuvo basada en los modelos de valores establecidos por otros autores (Pollay, 1983; Tse et al., 1989; Schwartz, 1994; Domínguez, 1995; Cheng y Schweitzer, 1996; Alameda, 2006), pero, al tratarse de modelos genéricos y empleados en televisión y prensa, no cum- 
plían los objetivos de este estudio, por lo que se buscaron modelos de valores establecidos por autores en la toma de decisión de compra de un coche (Byun, 2001; López et al., 2002; Chng et al., 2019). Unos valores que se analizaron y complementaron, estableciendo un modelo propio que aunaba las características de ambos.

Para definir los valores empleados en la publicidad automovilística, se realizó un trabajo de campo previo donde se le pidió a una muestra -constituida por 150 personas en edades comprendidas entre 18 y 60 años en el ámbito nacional español, seleccionadas a través de redes sociales- que categorizara en orden de importancia una serie de factores -obtenidos del modelo de Byun (2001) y López et al. (2002), principalmente- de los que se obtuvieron los siguientes (Tabla 5).

Tabla 5. Parámetros empleados para el análisis de valor

\begin{tabular}{|c|c|}
\hline Categorías & Descripción \\
\hline Seguridad & $\begin{array}{l}\text { Es el conjunto de todos aquellos elementos que contribuyen a proporcionar una mayor eficacia y estabilidad al } \\
\text { vehículo en marcha y, en la medida de lo posible, evitar un accidente. }\end{array}$ \\
\hline Precio & Valor monetario de un vehículo. \\
\hline Financiación & $\begin{array}{l}\text { Pago de un automóvil por cuotas. Se pacta un importe como pago de entrada del vehículo y una cuota mensual } \\
\text { durante un período que suele oscilar entre los tres y los cinco años. }\end{array}$ \\
\hline Promoción & $\begin{array}{l}\text { Conjunto de actividades cuyo objetivo es dar a conocer algo o incrementar sus ventas. Concretamente, este valor } \\
\text { se refiere a las ofertas y precios con descuento para incitar a la venta de un coche. }\end{array}$ \\
\hline Garantía & Cobertura legal y comercial que ofrece una marca. \\
\hline Ecología & $\begin{array}{l}\text { Avances para respetar el entorno y el medio ambiente. Las marcas más ecológicas son las que venden coches que, } \\
\text { fundamentalmente, tienen tamaños, potencias y consumos más contenidos. Vehículos sostenibles. }\end{array}$ \\
\hline Tecnología & Conjunto de instrumentos y procedimientos industriales. \\
\hline Confort & Bienestar o comodidad material que ofrece un coche. \\
\hline Espacio maletero & Capacidad que ofrece un vehículo para maletas o equipajes. \\
\hline Diseño & Boceto, bosquejo o esquema, la forma de un coche. Un ejemplo sería el diseño deportivo o clásico. \\
\hline Consumo & Gasto de combustible. \\
\hline Potencia & Poder o fuerza del motor. Se mide en caballos de vapor (CV) y kilovatios (KW). \\
\hline Infoentretenimiento & $\begin{array}{l}\text { Información + entretenimiento. Funciones del vehículo relacionadas con los sistemas que proporcionan infor- } \\
\text { mación -ordenador de a bordo, navegador, recomendaciones de conducción-, comunicación -telefonía, conec- } \\
\text { tividad a redes sociales desde el teléfono móvil- y entretenimiento -equipo de sonido, de vídeo y TV, reproductor } \\
\text { de imágenes-. }\end{array}$ \\
\hline Prestaciones & Servicios extras, tienen que ver con el equipamiento de un coche. \\
\hline Que cumpla legislación & $\begin{array}{l}\text { Este valor está relacionado con la normativa establecida en cuanto al nivel de emisiones de los coches y las res- } \\
\text { tricciones establecidas en cuanto al año de matriculación. }\end{array}$ \\
\hline
\end{tabular}

Fuente: elaboración propia 


\section{Resultados}

Siguiendo el mismo orden establecido en los objetivos, se plantean los resultados del análisis de contenido, la encuesta y la entrevista y, a partir de estos, se establece un modelo funcional para estudiar los criterios percibidos en la publicidad.

\subsection{Análisis de contenido}

En cuanto al análisis de contenido, se pone de manifiesto la importancia de los valores en la publicidad en función del contexto socioeconómico anterior y posterior a la crisis económica (Figura 4). Así, en 2008, seguramente debido al contexto de crisis económica, se reforzó el valor precio y promoción. Los anuncios presentaron ofertas, descuentos e, incluso, prestaciones incluidas en el precio durante un determinado periodo estacional. En 2018, sin embargo, se enfatizaron valores como el diseño, la financiación y la tecnología.

Figura 4. Comparativa valores 2008 vs. 2018

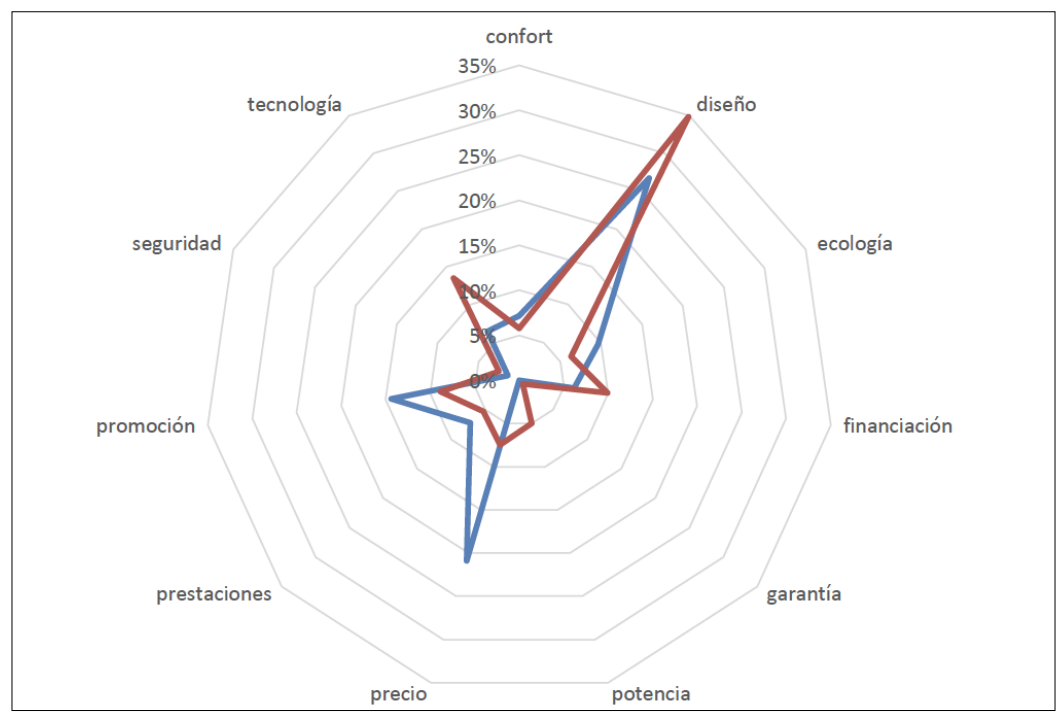

Fuente: elaboración propia

$\mathrm{Al}$ analizar la publicidad digital de los principales anunciantes por inversión en el medio Internet, Grupo Volkswagen, Grupo PSA-Peugeot-Citroën, Grupo Renault-Nissan-Mitsubishi, Ford y Grupo Hyundai-KIA concentraron la mayor inversión en el periodo 2007-2018, destacando por marcas SEAT, que representó un 14\% de la inversión total; Peugeot, un 11\%; Citroën y Volkswagen, ambos un $8 \%$ y Ford, un 7\%. Por segmentos, el 9-Todoterrenos fue el que más invirtió en Internet y el 4-Turismo medio alto, el que menos. La distribución de la inversión en el total de medios fue bastante similar a la inversión en el medio Internet. 
En lo que respecta al tipo de producto más anunciado en las creatividades de coches, las marcas invirtieron más en 2008 en publicitar los turismos 3 medios y en 2018, los todoterrenos. Concretamente, en 2008 la presencia de los turismos 3 medios fue del $27 \%$ y de los turismos 2 pequeños, del 25\%. Los todoterrenos, un 15\%, y los monovolúmenes, un $12 \%$. En 2018, los todoterrenos fueron los más anunciados, con un 49\%, le siguieron los turismos 3 medios, con un $27 \%$, y los turismos 2 pequeños, con un $18 \%$. Sin embargo, el argumentario de venta cambió en función de cada marca y tipo de turismo (Figura 5). No es lo mismo anunciar un coche tipo 1 micro diseñado para conducirlo por la ciudad, que un vehículo tipo todoterreno. En los turismos 5 lujo, por ejemplo, el diseño fue el valor más empleado por las marcas para anunciarlos, mientras que, en los turismos medio alto, el valor ecológico fue el más transmitido.

Figura 5. Comparativa por tipo de producto 2008-2018

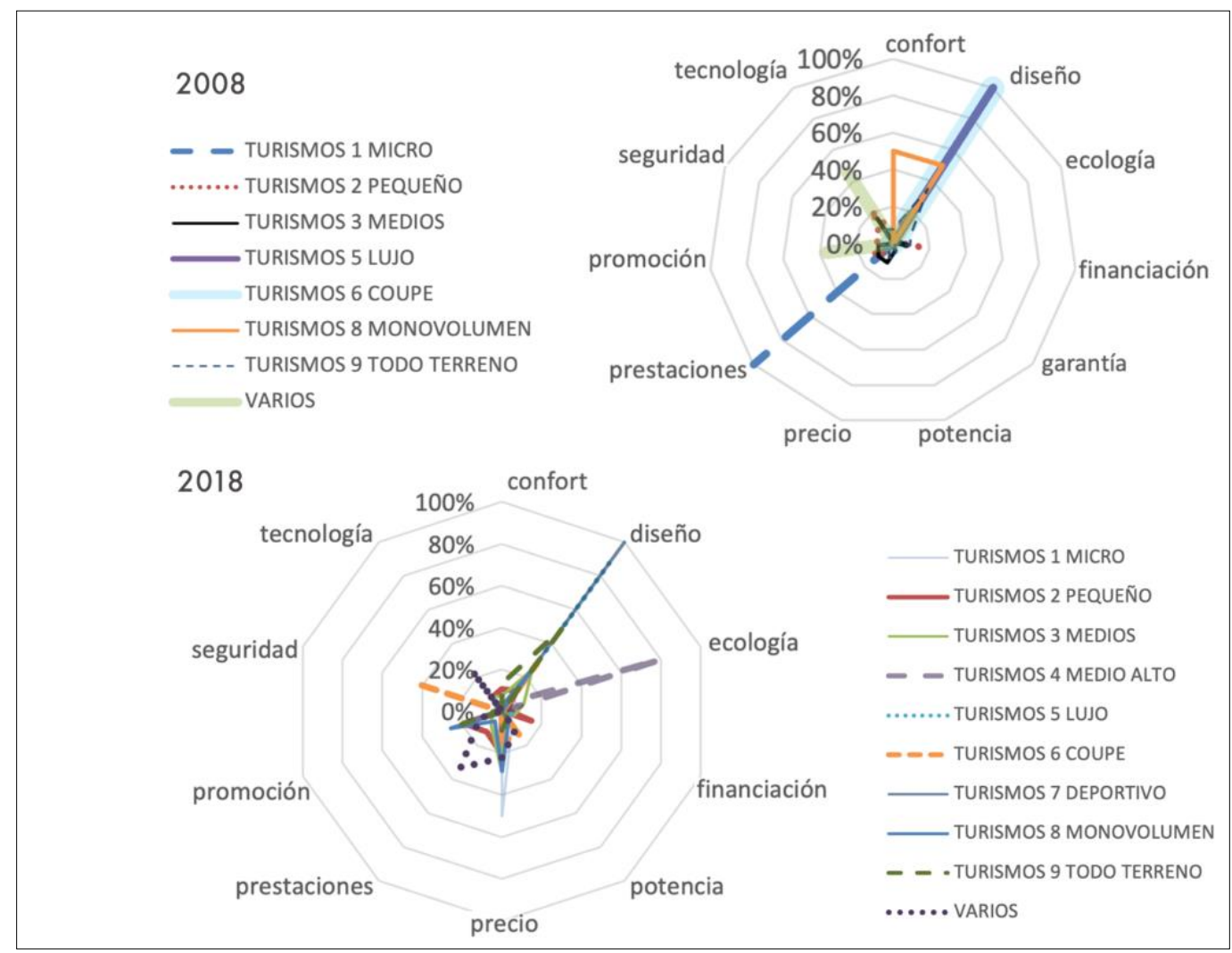

Fuente: elaboración propia a partir de datos obtenidos de Infoadex (2019) 
$\mathrm{Al}$ analizar en detalle la marca SEAT, que fue la que contó con mayor inversión publicitaria en el medio Internet durante el periodo seleccionado, se aprecia la distancia existente entre la publicidad de las marcas y los valores deseados por los consumidores (Figura 6). Así, los encuestados valoraron por encima del resto los valores de seguridad (4,3 sobre 5), consumo (4,0), precio (3,9) y un maletero espacioso $(3,9)$. Por debajo del resto, se situaron la promoción $(2,6)$, el infoentretenimiento $(2,6)$ y la financiación $(2,5)$. La publicidad realizada por esta marca utilizó valores que los encuestados puntuaron como menos deseados: la financiación, la promoción y la tecnología. Asimismo, emplearon otros que se valoraron positivamente, pero no los más valorados, como, por ejemplo, diseño $(3,5$ sobre 5$)$, prestaciones $(3,3)$ y potencia $(3,3)$.

Figura 6. Comparativa de valores deseados vs. publicitados en SEAT

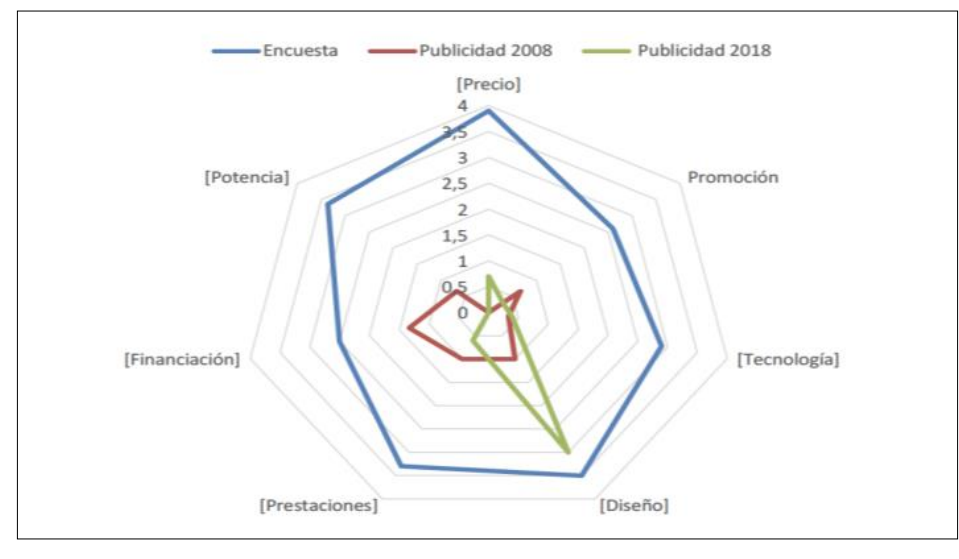

Fuente: elaboración propia

En 2008, la publicidad de marca incidió en la financiación, el diseño y las prestaciones, mientras que, en 2018, lo hizo en el diseño, las prestaciones y el precio.

\subsection{Encuesta}

Los encuestados tuvieron que valorar 14 criterios basados en una escala tipo Likert (seguridad; precio; financiación; garantía; promoción; confort; diseño; consumo; que sea ecológico; potencia; prestaciones; tecnología; que cumpla la legislación medioambiental; infoentretenimiento y maletero espacioso) de menor a mayor importancia desde 'nada relevante' a 'extremadamente relevante.' Los usuarios otorgaron mayor puntuación a la seguridad, seguida del consumo, que tenga un maletero espacioso y que cumpla la legislación medioambiental. Por otro lado, los atributos que valoraron con menos entusiasmo fueron la promoción, el infoentretenimiento y la garantía.

En relación al interés de los encuestados por la publicidad, un 51\% aseguró no fijarse en la publicidad, mientras que un 49\% afirmó hacerlo. El 51\% que no prestó atención a la publicidad está integrado por un $21 \%$ de los encuestados que afirmaron no tener coche propio y un $79 \%$ que sí lo posee. Del $49 \%$ de los encuestados que sí se fijaron en la publicidad, un 70\% fueron mujeres y un $30 \%$, hombres. Al ser preguntados por un recuerdo espontáneo de publicidad, se observa que el conocimiento sobre el sector de 
la muestra fue medio-bajo. Pocos encuestados pusieron ejemplos y, quienes lo hicieron, respondieron vagamente con ejemplos difusos, de hace años (por ejemplo, la campaña de BMW “¿Te gusta conducir?”). Solo un $10 \%$ citó un ejemplo de publicidad actual de Golf, y solo 1 persona reprodujo el claim: "Como un Golf no es un Golf”. Concretamente, un 55\% escribió un ejemplo e identificó el medio; el resto (un 45\%) no lo supo, no lo recordó o no supo dónde lo había visto. De este 55\% que contestó, un $45 \%$ nombró el medio televisión; un 7\%, el medio exterior (valla publicitaria); un 3\%, el medio impreso y un 1\%, la radio. Con respecto a la pregunta qué marca lo está haciendo bien en términos de publicidad y por qué, nombraron BMW, Mercedes, Toyota, Volkswagen, Kia y SEAT por delante del resto.

\subsection{Entrevistas a expertos}

Las variables precio y diseño fueron las más mencionadas por todos ellos. Los Responsables de Comunicación, Directores Generales y Presidentes de asociaciones del automovilismo diferenciaron dos tipos de clientes: el primero, el consumidor de marcas prémium, que buscan diseño y estatus principalmente. El segundo, el consumidor de marcas generalistas, que busca precio y que cumpla con las necesidades del cliente en cuanto a espacio, habitabilidad, consumo y movilidad. Asimismo, consideraron que ambos buscan seguridad e identificarse, de alguna manera, con la marca que van a utilizar. Asimismo, este grupo creyó que el cliente considera importante también el equipamiento, el confort, la fiabilidad y la conectividad.

Los periodistas, por el contrario, incidieron en la variable precio. Para estos, lo primero es el precio y, posteriormente, el diseño. Asimismo, prestaron especial atención al equipamiento en seguridad y a la calificación medioambiental.

El personal de venta fue el único que, además de mencionar todas estas variables, habló sobre la importancia del asesoramiento y del buen servicio postventa, diseño, precio y atención al cliente. "Primero el precio final y, casi a la misma altura, el trato y servicio".

\subsection{Modelo propuesto}

A partir de los resultados obtenidos en el análisis de contenido, las encuestas y las entrevistas en profundidad, se ha configurado un modelo de análisis propio basado en el diagrama de Venn para comparar visualmente entre sí los valores de la publicidad, tanto desde la perspectiva de los consumidores como de los expertos.

En el análisis de estos, se examinan los clústeres, comparando los criterios transmitidos en la publicidad con los valores deseados/demandados por los consumidores y con los factores más importantes en el proceso de compra, que emergen del discurso de los expertos entrevistados.

En las Figuras 7 y 8 se expone el resultado del modelo de análisis de valores realizado en los dos años estudiados: 2008 y 2018 en tres diagramas de Venn. El superior, de color naranja, presenta los valores que transmite la publicidad, identificados en una muestra representativa de 300 anuncios en 2008 y de 372 anuncios en 2018. Son los valores más empleados en la publicidad, tal y como se ha explicado en el apartado de metodología. El de la izquierda, de color verde, representa los valores demandados por los consumidores finales de las distintas marcas automovilísticas. Y, en último lugar, el conjunto de la derecha, de color azul, se refiere a los valores que los expertos del sector automovilístico entrevistados indicaron que son los prioritarios para los consumidores al tomar su decisión de compra. 
Figura 7. Comparativa valores en el año 2008

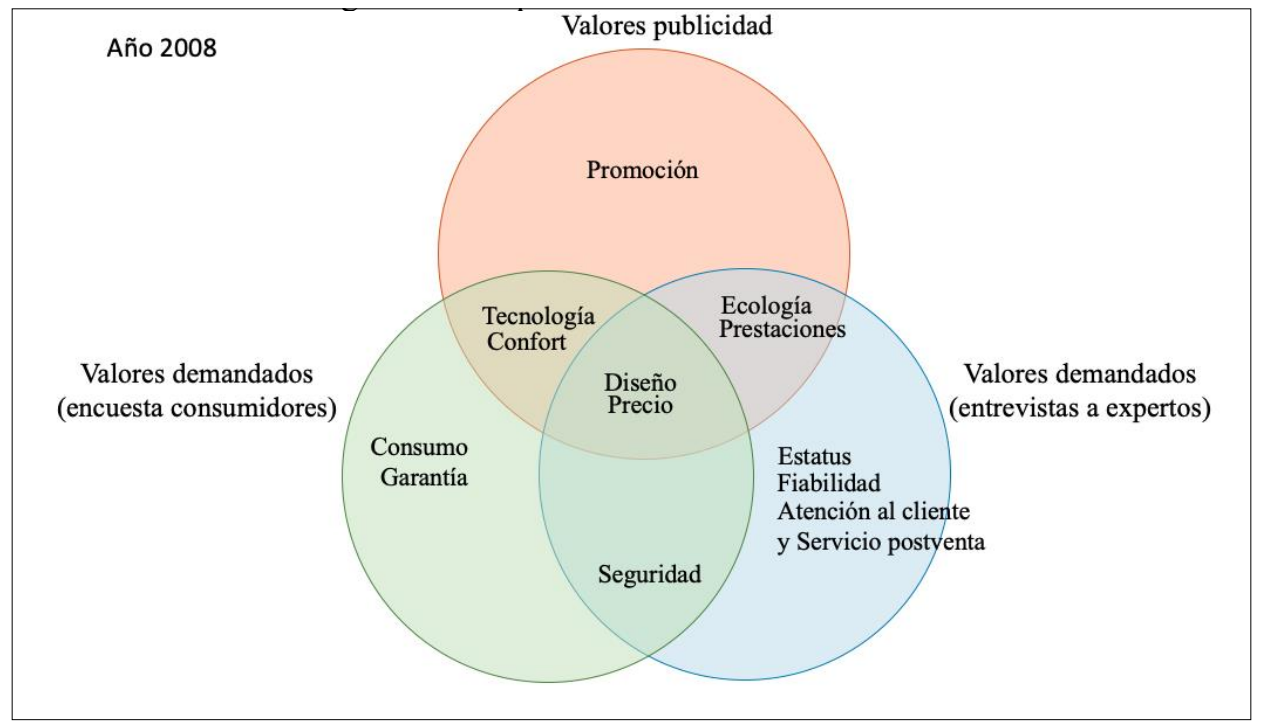

Fuente: elaboración propia

Figura 8. Comparativa valores en el año 2018

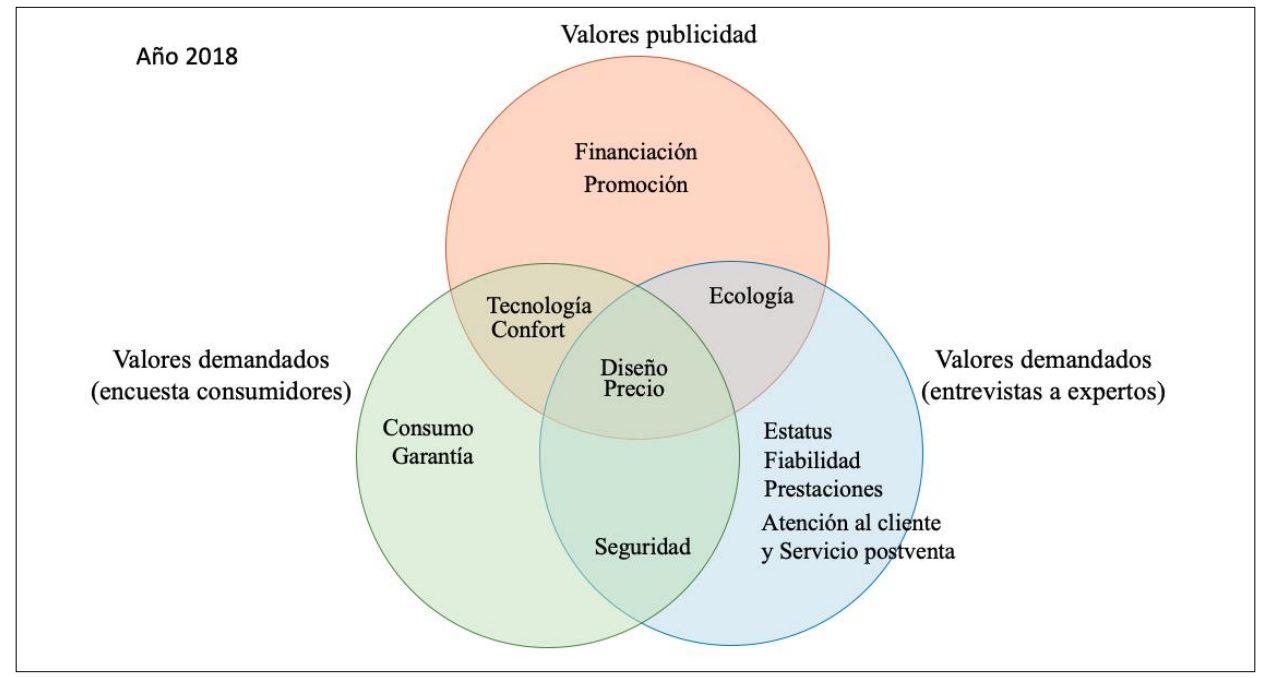

Fuente: elaboración propia 
Se observa, por tanto, una divergencia clara entre los valores que se están intentando transmitir, los que demandan los clientes y los que los expertos consideran más relevantes:

- Tanto en 2008 como en 2018, la publicidad transmitió que sus vehículos tenían una buena promoción -incluso en 2018, también financiación-, mientras que ni los clientes ni los expertos lo consideraron lo más relevante.

- En los dos años analizados, la publicidad refleja los valores de ecología y prestaciones, que también son importantes para los expertos, pero los consumidores no los valoraron de la misma forma. Algo parecido sucede con los criterios de confort y tecnología de los coches, que fueron demandados por los encuestados, pero los expertos no los consideraron aspectos decisivos para la compra.

- Los expertos consideraron la ecología; las prestaciones; el estatus; la fiabilidad; la atención al cliente y el servicio postventa como factores clave en la compra de un vehículo por parte de sus clientes. Valores que los propios consumidores no compartieron y, de los cuales, la publicidad sólo transmite la ecología -en 2008 y 2018- y las prestaciones -sólo en 2008-.

- Los consumidores valoran el consumo y la garantía, aspectos en los que los expertos no coincidieron y que la publicidad no refleja.

- La seguridad fue el aspecto más valorado, tanto por los consumidores a la hora de comprar un coche, como por los expertos. Sin embargo, la publicidad no la refleja en su discurso comercial.

- El diseño y el precio son los dos únicos criterios presentes en la publicidad de 2008 y 2018 que fueron valorados por los consumidores y que, a su vez, los expertos consideraron clave en la toma de decisión de compra de sus clientes.

\section{Discusión y conclusiones}

La investigación desarrollada ha permitido alcanzar el objetivo planteado inicialmente de analizar los valores desde 3 perspectivas: la publicidad, el consumidor y el experto. Aunque esta propuesta metodológica solo se haya empleado para analizar la publicidad de las marcas automovilísticas en Internet, se podría aplicar a la publicidad de otros sectores en Internet e, incluso, los coches en prensa y televisión, porque son muchas las posibilidades que ofrece la clasificación, pues se proponen categorías de análisis genéricas que se pueden ampliar y adaptar a cada caso de estudio, lo cual ayudaría a identificar los valores predominantes en los diferentes medios de comunicación. Una ventaja de utilizar Internet como medio publicitario es el mayor alcance que puede conseguir y, al ser un recurso digital, se adapta fácilmente a diversas plataformas. Además, la interactividad que puede conseguir ayuda a generar interés por parte de los usuarios y a que la marca tenga mayor visibilidad.

En cuanto a la percepción de valores, una conclusión obtenida es que hay diferencias entre los factores más relevantes por parte de los consumidores y los que transmiten las marcas en sus anuncios. Los valores que los consumidores demandan coinciden con los valores que la publicidad transmite sólo parcialmente en cuatro aspectos: diseño, precio, tecnología y confort. La publicidad también difunde financiación y promoción, pero los consumidores no los consideran muy relevantes. Los encuestados lo que más valoran es la seguridad; sin embargo, esto no se transmite en la publicidad. Igualmente, valoran el consumo y garantía, que tampoco está muy presente. Con base a lo analizado, se concluye que el papel de la publicidad no sólo es el de transmitir 
valores, sino orientar en ciertos intereses y preferencias de consumo, educar y marcar tendencias, generando una proyección internacional.

Las marcas comunicaron en 2008 valores como el precio, la promoción y la financiación, mientras que, en 2018, las marcas emplearon más el valor de la tecnología; por tanto, se deduce que las compañías adaptan su discurso en función del momento y las tendencias. En los últimos 10 años parecen surgir nuevas prioridades en la escala de valores que marcan la decisión de compra; por ejemplo, la publicidad realizada en 2008 da prioridad a unos valores y, en 2018, a otros diferentes. En los banners analizados de 2008 se repiten más los valores de diseño, precio, promoción, ecología, confort y tecnología -en este orden-, mientras que en 2018 los valores que más aparecen son los de diseño, tecnología, financiación, promoción y precio -en este orden-.

Asimismo, hay diferencias entre lo que los expertos del sector consideran que los clientes potenciales buscan y lo que estos realmente están buscando. Según los profesionales, en el proceso de compra los clientes lo que más tienen en cuenta es la seguridad, el diseño, el precio, la ecología, el estatus, la fiabilidad, las prestaciones, la atención al cliente y el servicio postventa. Sin embargo, los encuestados lo que más consideran es la seguridad, el diseño, el precio, la tecnología, el confort, el consumo y la garantía. Únicamente coinciden en el diseño, el precio y la seguridad y difieren en el resto. Esto denota que las marcas crean la publicidad tomando en cuenta las tendencias temáticas que van surgiendo; por ejemplo, actualmente es común observar en la publicidad de diferentes organizaciones su interés por el cuidado del medio ambiente o bien su aportación a la creación de un desarrollo sostenible. Por tanto, la decisión de compra del automóvil está condicionada por los nuevos valores que son coherentes con la evolución de los temas que preocupan a la sociedad actual, como son la ecología, la tecnología y que se cumpla con la legislación medioambiental. Igualmente, después de la crisis de la Covid-19, es posible que las marcas adapten sus diseños, haciendo especial hincapié en el habitáculo (filtros de aire que protejan ante el virus) y que esto se refleje en su comunicación, destacando nuevos elementos como el bienestar y la salud. Por todo ello, habrá que prestar especial atención a estos nuevos valores, que configuran las futuras líneas de comunicación.

\section{Agradecimientos}

Este artículo ha sido traducido al inglés por Charles E. Arthur.

\section{Referencias bibliográficas}

Alameda, D. (2006). Una nueva realidad publicitaria. La generación de valores corporativos en publicidad. Laberinto.

Bamberg, S., \& Möser, G. (2007). Twenty years after Hines, Hungerford, and Tomera: a new meta-analysis of psycho-social determinants of pro environmental behaviour. Journal of Environmental Psychology, 27(1), 14-25. https://doi.org/10.1016/j. jenvp.2006.12.002

Banerjee, S. (2010). Study on consumer buying behavior during purchase of a second car. Journal of Marketing \& Communication, 6(2), 4-13. https://bit.ly/3iC4g63.

Barbarossa, C., De Pelsmacker, P., \& Moons, I. (2017). Personal Values, Green Self-identity and Electric Car Adoption. Ecological Economics, 140, 190-200. https://doi.org/10.1016/j.ecolecon.2017.05.015 
Biedma, J. (1997). Valores de la publicidad y publicidad de los valores. Comunicar, 9, 61-68. https://doi.org/10.3916/C09-1997-09 Burgess, M., King, N., Harris, M., \& Lewis, E. (2013). Electric vehicle drivers' reported interactions with the public: Driving stereotype change?, Transportation Research Part F. Traffic Psychology and Behaviour, 17, 33-44. https://doi.org/10.1016/j.trf.2012.09.003 Byun, D. H. (2001). The AHP approach for selecting an automobile purchase model. Information \& Management, 38(5), $289-297$. https://doi.org/10.1016/S0378-7206(00)00071-9

Castelló, A., Ramos, I., \& Del Pino, C. (2013). El discurso publicitario en la crisis económica: nuevos valores y redes sociales. Historia y Comunicación Social, 18, 657-672. https://doi.org/10.5209/rev_HICS.2013.v18.43997

Castells, M. (2006). Internet y la sociedad red. Alianza.

Coad, A., de Haan, P., \& Woersdorfer, J. S. (2009). Consumer support for environmental policies: an application to purchases of green cars. Ecological Economics, 68(7), 2078-2086. https://doi.org/10.1016/j.ecolecon.2009.01.015

Codeluppi, V. (2007). El papel social de la publicidad. Pensar la Publicidad. Revista Internacional de Investigaciones Publicitarias, 1(1), 149-155. https://bit.ly/34FuhMz

Chng, S., White, M. P., Abraham, C., \& Skippon, S. (2019). Consideration of environmental factors in reflections on car purchases: Attitudinal, behavioural and sociodemographic predictors among a large UK sample. Journal of Cleaner Production, 230, 927-936. https://doi.org/10.1016/j.jclepro.2019.05.179

Cheng, H., \& Schweitzer, J. C. (1996). Cultural values reflected in Chinese and U.S. TV advertising. Journal of Advertising Research, $36(3), 27-45$.

Choo, S., \& Mokhtarian, P. (2004). What type of vehicle do people drive? The role of attitude and lifestyle in influencing vehicle type choice. Transportation Research Part A: Policy and Practice, 38(3), 201-222. https://bit.ly/38BRQXU

Domínguez, C. L. (1995). Publicidad y valores. Modelos de consumidor y análisis de valores en la publicidad televisiva española [Tesis Doctoral]. Universidad de Navarra.

García, M. (2018). ¿Te gusta conducir? Una mirada crítica desde la publicidad de coches y su retórica medioambiental. Didáctica de las Ciencias Experimentales y Sociales, 34, 91-104. https://doi.org/10.7203/DCES.34.11008

Gao, Y., Rasouli, S., Timmermans, H., \& Wang, Y. (2014). Reasons for not buying a car: a probit-selection multinomial logit choice model. Procedia Environmental Science, 22, 414-422. https://doi.org/10.1016/j.proenv.2014.11.039

Gunter, B., Furnham, A., \& Griffiths, S. (2000). Children's Memory for News: A comparison of three presentation media. Media Psychology, 2(2), 93-118. https://doi.org/10.1207/S1532785XMEP0202_1

Hafner, R. J., Walker, I., \& Verplanken, B. (2017). Image, not environmentalism: a qualitative exploration of factors influencing vehicle purchasing decisions. Transportation Research Part A: Policy and Practice, 97, 89-105. https://doi.org/10.1016/j. tra.2017.01.012

Hawkins, D. (1994). Electronic Advertising: On Online Information Systems. Online, 18(2), 26-39.

Heffner, R. R., Kurani, K. S., \& Turrentine, T. S. (2007). Symbolism and the adoption of fuel-cell vehicles. World Electric Vehicle Journal, 1(1), 24-31. https://doi.org/10.3390/wevj1010024 
Infoadex (2020). Mosaico. [base de datos]. https://bit.ly/3znVcck

Johnson, T. J., \& Kaye, B. K. (2000). Using is believing: The influence of reliance on the credibility of online political information among politically interested internet users. Journalism \& Mass Communication Quarterly, 77(4), 865-879. https://doi. org/10.1177/107769900007700409

Kahn, M. E. (2007). Do greens drive Hummers or hybrids? Environmental ideology as a determinant of consumer choice. Journal of Environental Economics and Management, 54(2), 129-145. https://doi.org/10.1016/j.jeem.2007.05.001

Lane, B. (2011). Market Delivery of Ultra-Low Carbon Vehicles in the UK An evidence review. RAC Foundation. https://bit. ly/2KWdz4A

Lane, B., \& Potter, S. (2007). The adoption of cleaner vehicles in the UK: exploring the consumer attitude-action gap. Journal of Cleaner Production, 15(11-12), 1085-1092. https://doi.org/10.1016/j.jclepro.2006.05.026

Lieven, T., Mühlmeier, S., Henkel, S., \& Waller, J. F. (2011). Who will buy electric cars? An empirical study in Germany. Transportation Research Part D: Transport and Environment, 16(3), 236-243. https://doi.org/10.1016/j.trd.2010.12.001

López, C., Fernández, K., \& Mariel, P. (2002). Índices de satisfacción del consumidor: Una aplicación de modelos de ecuaciones estructurales a la industria automovilística española. UPV/EHU.

Martín-Casado, T. G., Echazarreta, C., \& Vinyals, M. (2012). El relato audiovisual publicitario del siglo XXI. ¿Nuevos medios o nuevas formas de consumo audiovisual? Revista Comunicación, 10(1), 164-175. https://bit.ly/37NN3DB

McLuhan, M. (1994). The Extensions of Man. MIT Press.

Méndiz, A. (2007). Los valores en la publicidad: Un nuevo planteamiento ético y comercial. Icmedianet.

Núñez, M., Olarte, C., \& Reinares, E. (2008). Influencia de la publicidad en las tendencias sociales: Una aproximación exploratoria al mercado publicitario español. En J. Pindado, \& G. Payne (Coords.), Estableciendo puentes en una economía global (pp. 29-45). ESIC.

Núñez-Cansado, M., \& Martín-Requero, L. (2015). Autorregulación y creatividad publicitaria en el sector del automóvil en España: freno al tren verde. Vivat Academia, 132, 63-87. https://doi.org/10.15178/va.2015.132.38-87

Okazaki, S. (2002). La Estandarización Publicitaria de las Multinacionales Japonesas: Análisis de Contenido de la Publicidad Web en Japón, España y EE.UU. [Tesis Doctoral]. Universidad Autónoma de Madrid.

Peters, A., de Haan, P., \& Scholz, R. W., (2015). Understanding car-buying behavior: psychological determinants of energy efficiency and practical implications. International Journal of Sustainable Transportation, 9(1), 59-72. https://doi.org/10.1080/1556 8318.2012.732672

Pollay, R. W. (1983), Measuring the Cultural Values Manifest in Advertising. En J. A. Leigh, \& C. R. Martin, Jr. (Eds.), Current Issues and Research in Advertising (pp. 71-92). University of Michigan Press.

Pollay, R. W. (1986). The Distorted Mirror: Reflections on the Unintended Consequences of Advertising. Journal of Marketing, 50, 18-36. https://doi.org/10.2307/1251597 
Ramos, M., Delgado, M. T., \& Jiménez, G. (2007). Las nuevas estrategias de comunicación en el sector automovilístico: razón y emoción en la campaña de Audi. FISEC-Estrategias, 6(7), 23-45. http://hdl.handle.net/11441/28913

Rodríguez, Á., Montoya, N., Mas, LL., Morales, F., Lopes da Silva, E., Martins, G., Peixoto, M., \& Müller, K. (2013). Medición y evaluación de valores en contenidos audiovisuales desde un abordaje interdisciplinar. Prisma Social, 11, 158-204. https://bit. ly/3mMDHfB

Sandoval, M. (2007). Sociología de los valores y juventud. Ultima década, 15(27), 95-118. https://doi.org/10.4067/ S0718-22362007000200006

Schwartz, S. H. (1994). Are there universal aspects in the structure and contents of human values? Journal of Social Issues, 50(4), 19-45. https://doi.org/10.1111/j.1540-4560.1994.tb01196.x

Schwartz, S. H., \& Bilsky, W. (1990). Toward a Theory of the Universal Content and Structure of Values: Extensions and Cross-Cultural Replications. Journal of Personality and Social Psychology, 58(5), 878-891. https://doi.org/10.1037/0022-3514.58.5.878

Steg, L. (2005). Car use: lust and must. Instrumental, symbolic and affective motives for car use. Transportation Research Part A: Policy and Practice, 39(2-3), 147-162. https://doi.org/10.1016/j.tra.2004.07.001

Steg, L., Vlek, C., \& Slotegraaf, G. (2001). Instrumental-reasoned and symbolic-affective motives for using a motor car. Transportation Research Part F: Traffic Psychology and Behaviour, 4(3), 151-169. https://doi.org/10.1016/S1369-8478(01)00020-1

Trespalacios, J. A., Vázquez, R., \& Bello L. (2005). Investigación de mercados y estrategias de marketing. Civitas.

Tse, D. K., Belk, R. W., \& Zhou, N. (1989). Becoming a consumer society: a longitudinal and cross-cultural content analysis of print ads from Hong Kong, the People's Republic of China, and Taiwan. Journal of Consumer Research, 15(4), 457-472. https:// doi.org/10.1086/209185

Victoria, J. S. (2005). Reestructuras del sistema publicitario. Ariel.

Yoon, S., \& Kim, J. (2001). Is the Internet More Effective Than Traditional Media? Factors Affecting the Choice of Media. Journal of Advertising Research, 41(6), 53-60. https://doi.org/10.2501/JAR-41-6-53-6 